
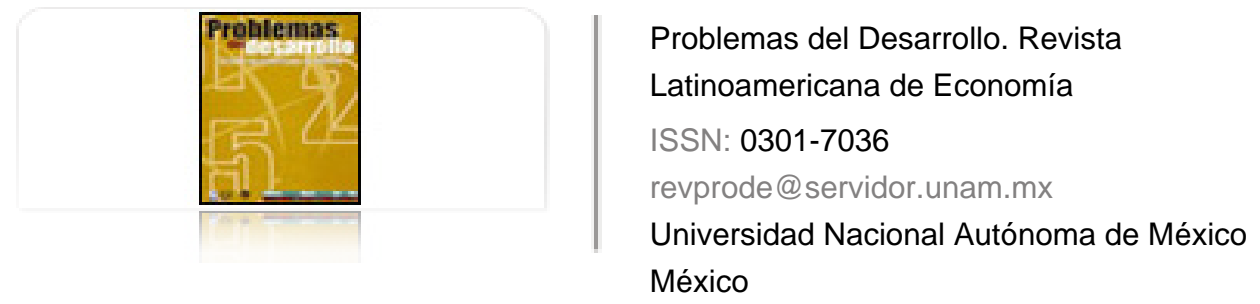

Gaulard, Mylène

LA DÉSINDUSTRIALISATION BRÉSILIENNE: UNE LECTURE MARXISTE

Problemas del Desarrollo. Revista Latinoamericana de Economía, vol. 41, núm. 161, abril-junio, 2010, pp. 81-107

Universidad Nacional Autónoma de México

Distrito Federal, México

Disponible en: http://www.redalyc.org/articulo.oa?id=11820105004

Comment citer

Numéro complet

- Plus d'informations de cet article

Site Web du journal dans redalyc.org

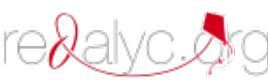

Système d'Information Scientifique Réseau de revues scientifiques de l'Amérique latine, les Caraïbes, l'Espagne et le Portugal Projet académique sans but lucratif, développé sous l'initiative pour l'accès ouverte 


\title{
LA DÉSINDUSTRIALISATION BRÉSILIENNE: UNE LECTURE MARXISTE
}

\author{
Mylène Gaulard*
}

Fecha de recepción: 9 de marzo de 2010. Fecha de aceptación: 12 de abril de 2010.

\section{Résumé}

La financiarisation de l'économie brésilienne est souvent montrée du doigt comme la principale responsable du ralentissement du processus d'accumulation. Ainsi, des taux d'intérêt élevés incitent davantage à se procurer des bons du trésor qu'à investir dans la sphère productive. Néanmoins, la fin du «Miracle économique» et la crise de la décennie 1980 peuvent être analysées comme des conséquences d'une baisse $d u$ taux de profit dans l'industrie. Actuellement, cette évolution explique en grande partie l'orientation massive des investisseurs vers la sphère financière. La financiarisation est donc ici étudiée comme une conséquence, et non comme une cause, des difficultés rencontrées dans la sphère productive.

Mots-clés: Brésil, désindustrialisation, finance, crise, profit.

* Doctora en Economía Internacional, Université Paris I-Panthéon Sorbonne. Es Profesora-investigadora de economía internacional ISTOM, escuela de ingeniería en agro-desarrollo internacional. Correo electrónico: gaulard@ netcourrier.com 


\section{Resumen}

La financiarización de la economía brasileña con frecuencia es señalada como la mayor responsable de la disminución de la velocidad del proceso de acumulación en ese país. Así, tipos de interés muy elevados, particularmente con el fin de financiar la deuda pública, incitan más a adquirir bonos del tesoro que a invertir en la esfera productiva. Sin embargo, la evolución del índice de provecho en esta última también explica la relación de los empresarios con la inversión. En efecto, el fin del "milagro económico" y la crisis de la década de 1980 pueden ser analizados como consecuencias de un descenso del índice de provecho en la industria. Esta evolución explica en gran parte la orientación masiva de los inversionistas hacia la esfera financiera. Por esta razón, la financiarización es estudiada aquí como una consecuencia, y no como una causa, de las dificultades encontradas en la esfera productiva.

Palabras clave: Brasil, desindustrialización, finanzas, crisis, provecho.

\section{Abstract}

The financialization of the Brazilian economy is often criticized as being responsible of the slowdown of capital accumulation. Indeed, very high interest rates foster investors to get more Treasury bonds rather than investing in the productive area. Nevertheless, the end of the "Economic Miracle" and the crisis of the 1980s can be analysed as consequences of the falling rate of profit in industry. Nowadays, this evolution explains a great part of the orientation of investors towards finance. The financialization is studied here as a consequence, and not as a cause, of the difficulties met in the productive apparatus.

Keywords: Brazil, desindustrialization, finance, crisis, profit.

\section{Resumo}

A financiarização da economia brasileira é assinalada frequentemente como a maior responsável da diminuição de velocidade do processo de acumulação nesse país. Nesse sentido, taxas de juros muito elevados, particularmente destinados a financiar a divida pública, incitam mais a proporcionar bônus do tesouro do que a investir na esfera produtiva. No entanto, a evolução do índice de aproveitamento nesta última também explica a relação dos empresários com os investimentos. Na realidade, o fim do "milagre econômico" e a crise da década de 1980 podem ser analisados como consequencias de uma diminuição do índice de aproveitamento na indústria. Esta evolução explica em grande parte a orientação massiva dos investidores para a esfera financeira. Por esta razão, a financiarização estuda-se aqui como uma consequência, e não como uma causa, das dificuldades encontradas na esfera produtiva.

Palavras-chave: Brasil, desindustrialização, finanças, crise, aproveitamento. 


\section{Introduction}

fin de mieux comprendre le fonctionnement de l'économie mondiale, et notamment le rôle de la finance dans le déclenchement des crises, il est essentiel de s'interroger sur le processus actuel de financiarisation de nos économies. Le Brésil est confronté à un tel processus depuis le début des années 1980. La financiarisation s'y présente comme la hausse exponentielle des revenus provenant de la détention des différents types d'actifs financiers (actions, obligations, créances), évolution attribuée aux taux d'intérêt élevés observés dans le pays. Il s'agit en fait d'un nouveau régime d'accumulation du capital au sein duquel le processus de production est subordonné aux hautes exigences de rentabilité dictées par les institutions financières, régime parfaitement analysé dans le cas des pays développés par des économistes marxistes ( $c f$. Chesnais, De Brunhoff, Duménil et Husson, 2006), régulationnistes ( $c f$. Boyer, 2000; Aglietta, 2007) ou post-keynésiens ( $c f$. Stockhammer, 2004), et ce depuis les an-

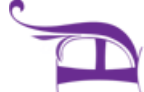
nées 80. Dans la littérature économique brésilienne, on insiste souvent sur le fait que cette financiarisation est à l'origine du ralentissement de l'accumulation, autrement dit, d'une stagnation de la formation brute de capital fixe. Nous verrons pourtant que la rente financière tend à diminuer depuis le début de la décennie 2000, et néanmoins, le taux d'investissement demeure toujours aussi faible. Il sera donc nécessaire de proposer une nouvelle explication au ralentissement du processus d'accumulation. Notamment, nous considérerons dans la deuxième partie de cet article que celui-ci est davantage lié à la baisse du taux de profit dans l'appareil productif, elle-même causée par l'augmentation de la composition organique du capital, c'est-à-dire, selon une approche marxiste, du rapport entre le capital constant (valeur des moyens de production, bâtiments, machines, matières premières) et le capital variable (valeur de la force de travail) utilisés dans le processus de production. Nous rejoindrons en cela la thèse développée par Karl Marx dans le troisième livre du Capital.

\section{La financiarisation de l'économie brésilienne}

Nous verrons dans un premier temps les faits et arguments qui soutiennent la thèse selon laquelle la financiarisation serait responsable du ralentissement du processus d'accumulation, en envisageant la faiblesse du taux d'investissement brésilien comme son effet principal.

\section{L'analyse du taux d'investissement brésilien: un investissement trop faible}

Depuis la décennie perdue des années 1980, le Brésil est très souvent stigmatisé pour son défaut d'investissement. Celui-ci stagne entre 15 et $18 \%$ du PIB. Ce serait même 
Figure 1

Taux de formation brute de capital fixe au Brésil à prix courants et constants (1972-2009)

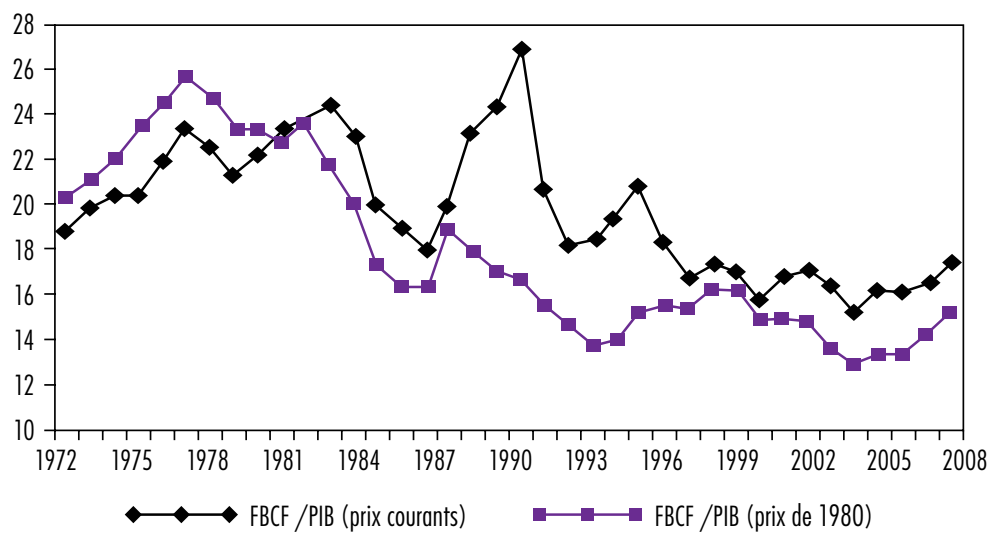

Source: IPEADATA, graphique réalisé par l'auteur.

pire: selon Feu (2004b) ou le IEDI (2007), la supposée stagnation (et non la baisse) du taux de FBCF entre 1998 et 2004 est due au fait que le coût du capital fixe ne cesse de s'élever. En effet, si au contraire, on considère des prix constants pour le capital fixe (prix de 1980, d'après la figure ${ }^{\circ} 1$ ), le taux de FBCF, après avoir fortement chuté de 1977 à 1994, aurait continué à diminuer après 1998 (de 16\% en 1998, à $13 \%$ en 2004). Certes, depuis 2004, le taux de FBCF (aussi bien celui à prix courants que celui à prix de 1980) augmente légèrement, mais il reste cependant inférieur à 20 pour cent.

Cette faiblesse du taux de formation brute de capital fixe s'accompagne d'une diminution relative du poids de l'industrie nationale dans la création de valeur ajoutée. Ainsi, l'industrie de transformation représente moins de 18\% du PIB en 2005 (contre $41 \%$ pour la Chine), alors que cette part était de 32\% en 1986 (IEDI, 2005). Le Brésil est entré dans une phase de «désindustrialisation», phénomène qui s'observe aussi bien au niveau de sa structure productive que de ses exportations. Alors qu'en 1994, la part des produits manufacturés dans les exportations brésiliennes était de 57,3\%, ces produits ne représentent aujourd'hui qu'un peu moins de 50\% des exportations.

Cette désindustrialisation s'observe également en décomposant le capital fixe qui est utilisé chaque année. En effet, le capital fixe n'est pas forcément du capital productif participant au processus d'accumulation, car le taux de FBCF comprend aussi tout ce qui est investissement résidentiel, et ce dernier occupe une part croissante du stock de capital fixe (figure n²). En 1979, les machines et biens d'équipement parti- 
cipaient pour $30 \%$ du total du capital fixe productif, alors que cette part n'est plus que de 17\% en 2004 (Bruno, 2005). On constate en effet l'importance grandissante des constructions résidentielles et industrielles. Le taux de formation brute de capital fixe directement destiné au processus d'accumulation (achat de biens d'équipement) est donc encore plus faible que le taux de FBCF qui est habituellement présenté.

Selon un récent rapport du BNDES (Teixeira, 2007), l'industrie de transformation et l'infrastructure nationales représentaient $52 \%$ de tout l'investissement réalisé dans l'économie brésilienne au cours des années 70, et en 2006, cette part n'est plus que de 29,6\%. L'espace laissé par ces deux « secteurs » de l'économie est occupé par les services, ces derniers étant passés de $9 \%$ de la formation brute de capital fixe dans les années 70, à 30\% aujourd' hui. La construction résidentielle a aussi pris une place importante, et elle représente actuellement plus de $20 \%$ de la FBCF.

Figure 2

Part de la construction résidentielle et des biens d'équipement dans le stock de capital fixe au Brésil, en pourcentage (1953-2008)

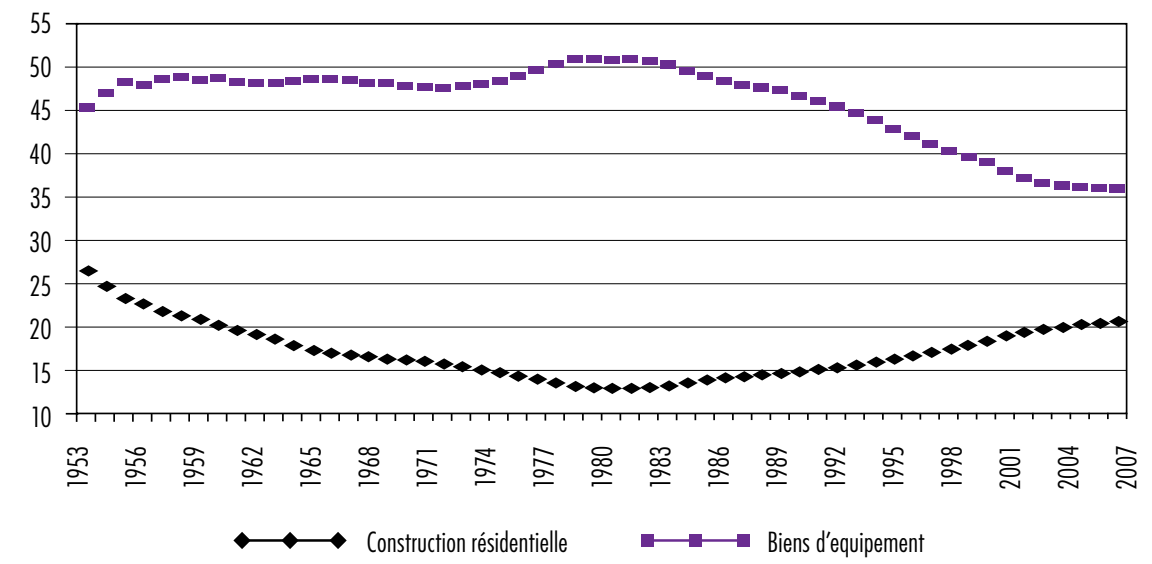

Source: IPEADATA, graphique réalisé par l'auteur.

Le Brésil présente donc un faible taux d'accumulation, et l'explication la plus souvent avancée pour appréhender ce phénomène repose sur l'analyse de la finance brésilienne. On assisterait dans ce pays à un processus de «financiarisation» de l'économie. Les rentiers accapareraient une part de plus en plus importante de la richesse nationale, du fait de taux d'intérêt très élevés, et ce aux dépens du processus d'accumulation. Cette financiarisation de l'économie serait donc responsable de la stagnation du taux d'investissement. 
La financiarisation de l'économie, responsable du ralentissement du processus d'accumulation?

Selon l'économiste Miguel Bruno (2005), la place des rentiers s'élargirait aux dépens de l'investissement productif en raison de l'insertion du Brésil dans le «processus actuel de globalisation»: les normes internationales ${ }^{1}$ imposeraient aujourd'hui, selon Bruno, de forts taux d'intérêt, et l'élévation de la charge de la dette diminuerait alors les profits dont bénéficient les investissements productifs, d'où le poids croissant de la finance et la financiarisation de l'économie brésilienne. Les taux d'intérêt brésiliens sont effectivement parmi les plus hauts du monde. Le Selic, taux d'intérêt de court terme, atteint 8,75\% en janvier 2010.

Ce qui explique la hauteur de ces taux, c'est la politique du gouvernement brésilien: ce dernier cherche à transformer sa dette extérieure, due à des agents économiques étrangers (souvent en dollars), en dette intérieure, due à des agents résidant dans le pays (en reals), en rendant très attractifs les bons du Trésor brésiliens. Afin de rembourser la dette publique externe, des bons du Trésor sont mis en circulation, qui sont d'autant plus attrayants que leurs taux d'intérêt sont élevés. Aujourd'hui, le paiement du service de la dette est assuré par l'émission permanente de ces bons. Cette dernière est donc à l'origine d'une forte augmentation de la rente financière (flux total des intérêts et dividendes perçus par le système bancaire et financier brésilien), et d'une concentration de la richesse financière.

Selon Bruno (2005), cette financiarisation de l'économie remonterait aux décennies des années 60 et 70; en 1964, le Programme d'Action Economique du Gouvernement (PAEG) établissait un dispositif de correction monétaire qui indexait la valeur des actifs financiers sur l'inflation. Ce dispositif permettait de réduire les risques de pertes financières, et de promouvoir ainsi le crédit pour accroître la consommation et l'investissement durant la période du «Miracle économique». Par ailleurs, à cette

1 Pourtant, dans son dernier ouvrage, Bresser-Pereira (2007: 197) insiste bien sur le fait que l'évolution des taux d'intérêt au Brésil ne reflète même plus l'évolution des primes de risque établies pour ce pays au niveau international. En effet, depuis plus de 10 ans, ces primes de risque ont fortement chuté. Aujourd'hui, seule la peur de l'inflation pousse la banque centrale à maintenir, ou à élever les taux d'intérêt (à la mi-avril 2008, les taux sont passés de 11,25 à $11,75 \%$, puis à $12,25 \%$ en juin, car en février 2008, les prévisions d'inflation pour l'année atteignaient $10 \%$, le plus haut niveau depuis 2005). Il n'en reste pas moins que cette peur est peu fondée, car il n'existe pas de tension sur les capacités de production, et la hausse des prix n'est donc pas due à une demande excessive qu'il serait nécessaire de freiner. Cette évolution, observée dans le monde entier jusqu'à l'été 2008, était plutôt le résultat d'une forte augmentation du prix des matières premières, laquelle ne pouvait être freinée par la hausse des taux d'intérêt. 
époque l'endettement de l'Etat brésilien paraissait nécessaire pour soutenir le processus d'accumulation.

Effectivement, ce dernier a longtemps assumé le rôle de la bourgeoisie en stimulant le processus d'accumulation. Dans ce pays, cette classe sociale n'était pas suffisamment développée lors des premiers pas dans le processus d'industrialisation, et l'Etat devenait indispensable pour la remplacer. L'accumulation fut donc stimulée par la création d'entreprises nationales et la prise en main d'une production peu rentable par l'Etat. Des produits bon marché étaient ainsi fournis aux entreprises privées, nationales et étrangères, ce qui encourageait l'essor de la bourgeoisie brésilienne. La contrepartie en fut l'accroissement de la dette publique externe dans les années 70 . Au cours de cette décennie, on voit en effet les revenus des pays pétroliers s'élever et

Figure 3

Dette publique nette, interne et externe, au Brésil (en \% du PIB), de 1991 à 2007

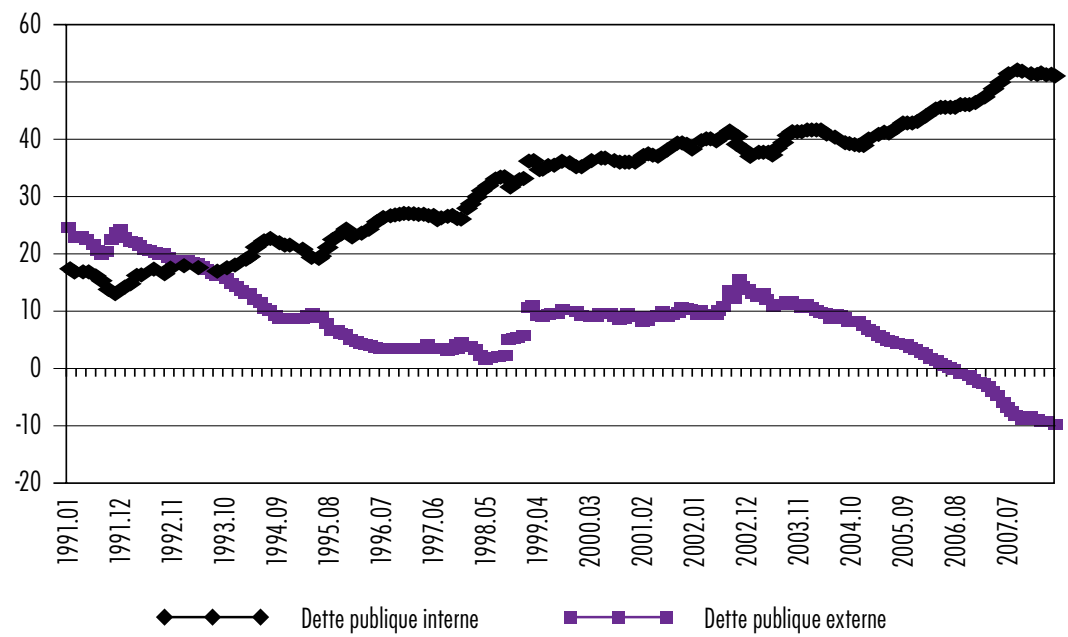

Source: IPEADATA, graphique réalisé par l'auteur.

être investis sous forme de prêts dans des pays en développement comme le Brésil.

Ce n'est qu'avec la crise des années 1980 que nous assistons à un processus d'«autonomisation de la dette», cette dernière ne servant plus à stimuler l'accumulation du capital, mais à accroître uniquement le revenu des plus riches: la dette de l'Etat brésilien ne cesse alors de se creuser, non pas pour financer de nouveaux investissements, mais pour faire face au service de la dette. Comme le révèle la figure 3 ci-dessus, les taux d'intérêt élevés permettent aux classes aisées de la population brésilienne de profiter des bons du Trésor pour grossir leurs revenus: alors que pendant longtemps le pays fit essentiellement appel à des capitaux étrangers, la dette 
actuelle est majoritairement interne, financée par l'épargne nationale qui n'est alors plus disponible pour financer des investissements productifs. Au Brésil, la dette publique externe est ainsi transformée en dette interne: l'Etat n'alimente plus ses finances sur les marchés internationaux, mais en émettant régulièrement des bons du Trésor au niveau national. Il n'en reste pas moins que les capitaux servant à acheter ces bons peuvent également provenir de l'extérieur: ils sont empruntés à l'étranger par les nationaux, ou bien ils proviennent directement d'investisseurs étrangers. Les bons du trésor brésiliens sont donc à l'origine d'un processus de financiarisation de l'économie nationale.

Cette politique serait à l'origine d'une forte augmentation de la rente financière, et de sa concentration. Les cinq plus grandes banques brésiliennes détiendraient $69 \%$ des profits du système bancaire, et $50 \%$ de ces profits proviendraient de la dette publique (Boito Junior, 2006). En 2006, seulement 15000 familles possédaient $80 \%$ des titres publiques fédéraux, ce qui met en évidence la forte concentration des revenus qu'engendre cette financiarisation de l'économie qui, de plus, a évidemment un impact sur le taux de profit net, et de là, sur l'investissement productif.

Selon Reinaldo Gonçalves (2006), les forts taux d'intérêt observés depuis 1995 participent à un mécanisme de transfert des revenus vers le secteur financier de l'économie. Le taux moyen d'investissement, de 1980-1994 à 1995-2004, est ainsi passé de 21,3\% à 19,3\% du PIB, alors que le taux d'intérêt moyen (de court terme) se serait élevé de $3,8 \%$ à 12,3\%. Les profits réalisés dans la finance se font au détriment des profits demeurant dans le secteur productif, et ce en raison de la hauteur des taux d'intérêt réclamés par les créanciers. Ainsi, le taux d'intérêt auquel s'effectuent les emprunts des entreprises et des particuliers dépasse $30 \%$, et le spread bancaire (différence entre le taux d'intérêt de court terme et le taux réclamé par les banques)

Tableau 1

Taux d'intérêt moyen au Brésil pour différentes opérations bancaires

\begin{tabular}{|l|c|}
\hline & Taux d'intérêt moyen \\
\hline Chèque spécial & $149 \%$ \\
\hline Crédit personnel & $48 \%$ \\
\hline Acquisition de biens-véhicules & $27 \%$ \\
\hline Acquisition de biens-autres & $55 \%$ \\
\hline Général & $38 \%$ \\
\hline
\end{tabular}

Source: Banco Central do Brasil. 
est supérieur à $20 \%$, ce qui rend le crédit extrêmement coûteux.

En conséquence, le taux de profit moyen du capital financier diminuait seulement d'une moyenne de 22,4\% entre 1980 et 1994, à 19,4\% (baisse de 13\%) entre 1995 et 2004, alors que le taux de profit moyen du capital productif (correspondant ici à la relation entre les profits nets réalisés et les fonds propres de l'appareil productif) passait de $8,2 \%$ à 5,6\% (baisse de 32\%) au fil de ces deux périodes (Gonçalves, 2006). La chute du taux de profit pour le capital financier est donc beaucoup moins importante que la chute du taux de profit net (profit qui reste à la disposition des entrepreneurs une fois que les prélèvements financiers ont été soustraits au profit brut). Enfin, les taux d'intérêt élevés observés au Brésil pénalisent l'investissement non seulement en augmentant la charge de la dette des entreprises, mais également en limitant l'offre de crédit: le volume du crédit correspondait à $80 \%$ du PIB en 1976, contre 35\% en 2008 (Gaulard, 2008).

\section{Nécessité de relativiser la responsabilité de la finance dans le processus}

\section{de désindustrialisation}

Le faible taux d'investissement brésilien s'expliquerait donc par la présence de taux d'intérêt très excessifs. Le pays connaîtrait actuellement une période exceptionnelle de «financiarisation» de l'économie due à l'essor international des marchés financiers (Bruno, 2005). Cependant, cette explication avancée par Miguel Bruno ainsi que par de nombreuses études réalisées sur le Brésil actuel (Gonçalves, 2006; Salama, 2000) ne peut appréhender que la situation présente. Or, déjà en 1982, Maria de Conceição Tavares observait que «des taux d'intérêt élevés s'accompagnent de l'expansion de la dette externe, dette dont les titres deviennent encore plus attrayants pour placer les ressources oisives des grandes entreprises et des banques» $»^{2}$ (apud Serra, 1982: 137). Dès la fin des années 1970, l'économie brésilienne faisait donc face à un affaiblissement de son taux d'investissement en raison de l'importance des taux d'intérêt. Cette époque n'était pourtant pas encore caractérisée par un essor de la finance mondiale.

Par ailleurs, comme nous le révèle la figure 4 , les taux d'intérêt réels auraient actuellement plutôt tendance à baisser (le taux réel de court terme passant de $24 \%$ en 1999, à 7\% en 2008), ce que Miguel Bruno peut difficilement expliquer par sa théorie. De même, à l'échelle mondiale, l'abondance d'épargne engendre des taux d'intérêt extrêmement bas. La rente croissante des pays pétroliers (due à l'augmenta-

2 Traduction de l'auteur. 
Figure 4

Taux d'intérêt réel de court terme Selic (1996-2008)

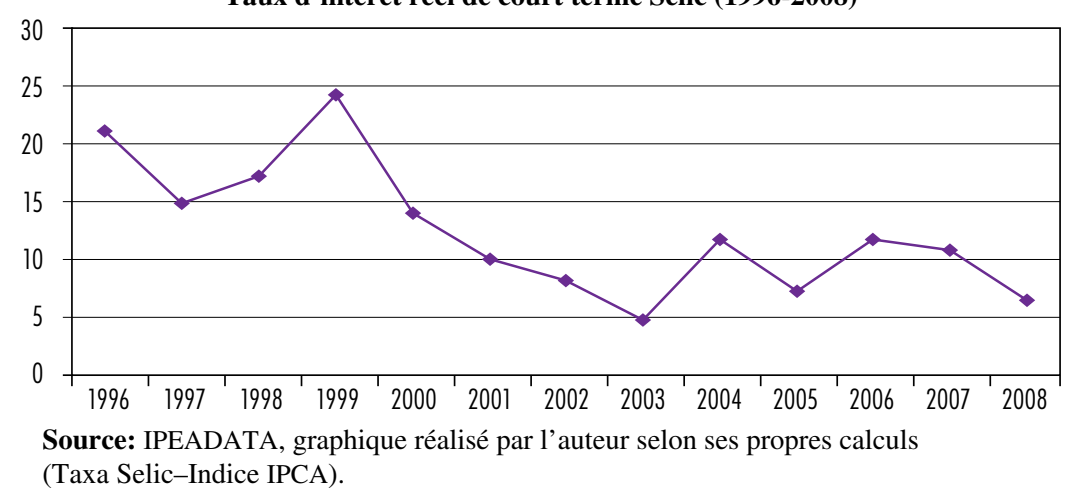

tion du prix du pétrole jusqu'à l'été 2008), la forte épargne des pays asiatiques (due au ralentissement de l'investissement dans ces pays, excepté la Chine, depuis la crise de 1997), ainsi que le faible taux d'investissement que connaissent l'ensemble des pays développés depuis la fin des Trente Glorieuses, sont à l'origine d'une épargne surabondante (Brender et Pisani, 2007). Cette épargne sert notamment à financer l'endettement américain, mais peut aussi expliquer l'essor sans précédent du secteur financier. Concevoir le processus de financiarisation actuel comme le résultat du niveau élevé des taux d'intérêt n'est donc pas une interprétation pertinente. Au contraire, les taux d'intérêt n'ont jamais été aussi bas au cours des 15 ou 20 dernières années, aussi bien au Brésil que dans le reste du monde.

Les taux d'intérêt payés chaque année par l'Etat brésilien pour rembourser sa dette sont en baisse depuis 2003 (passant de 8,99\% du PIB en juin 2003, à 6,06\% en mai 2008). Une telle évolution s'explique essentiellement par la diminution du taux d'intérêt de court terme ou Selic, celui-ci passant de 24\% en 2003 (avec une inflation de $9 \%$, le taux d'intérêt réel atteint tout de même 15\%), à 8,75\% en janvier 2010 (taux d'intérêt réel de 4,20 pour cent).

De même, l'excédent brut d'exploitation, comprenant les taux d'intérêt, les dividendes et les profits nets, a plutôt tendance à stagner depuis 1999, et même à diminuer depuis 2005 (Gaulard, 2008). Cet excédent brut d'exploitation ne constitue que 16\% du revenu national brut lorsqu' on en soustrait la formation brute de capital fixe, et les revenus de propriété, si souvent décriés, ne représentent «que» 3,5\% de ce revenu national, intérêts et dividendes compris. Il est vrai que les dividendes ont beaucoup augmenté depuis 2002, mais sur l'ensemble des revenus de propriété, cette hausse ne 
Figure 5

Intérêts de la dette publique brésilienne, en pourcentage du PIB (2000-2008)

Taux d'intérêt-gouv.

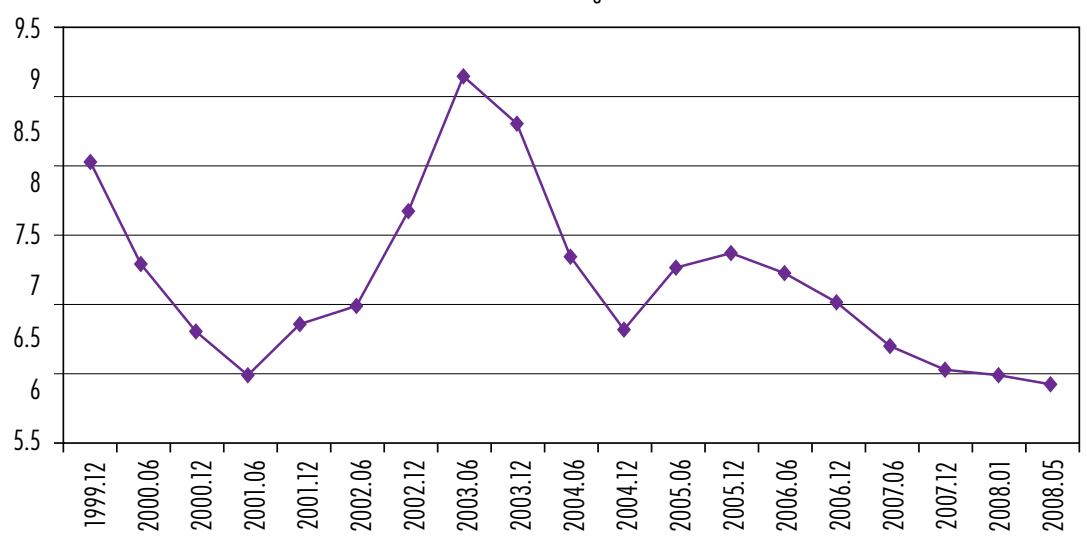

$\longmapsto$ Serie

Source: Banco Central, graphique réalisé par l'auteur.

compense pas l'amoindrissement des revenus liés aux taux d'intérêt.

\section{Figure 6}

Revenus de la propriété ${ }^{3}$ au Brésil en pourcentage du revenu national brut (1997-2007)

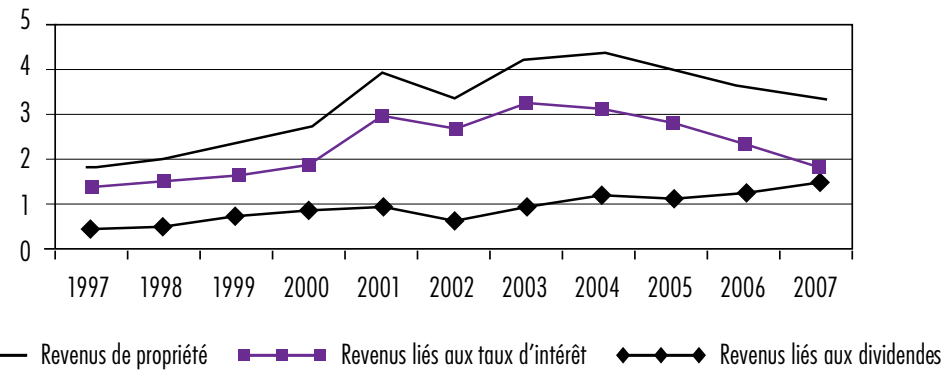

Source: IPEADATA, graphique réalisé par l'auteur (intérêts et dividendes demeurant dans le pays).

En raison de cette baisse des taux d'intérêt, le crédit semble également se trouver sur une pente ascendante depuis 2003, mais cela ne se traduit pas pour autant par une véritable reprise du processus d'accumulation. Comme nous l'avons vu précédemment, la formation brute de capital fixe continue d'être inférieure à $20 \%$ du PIB, alors que, comme nous le révèle le figure 7 , les opérations de crédit sont passées de $24 \%$ à $36 \%$ du PIB entre 2003 et 2008.

3 Revenus que reçoivent les propriétaires d'actifs financiers (taux d'intérêt pour les détenteurs d'obligations, et dividendes pour les actionnaires). 
Figure 7

Opérations de crédit effectuées au Brésil en pourcentage du PIB (2000-2008)

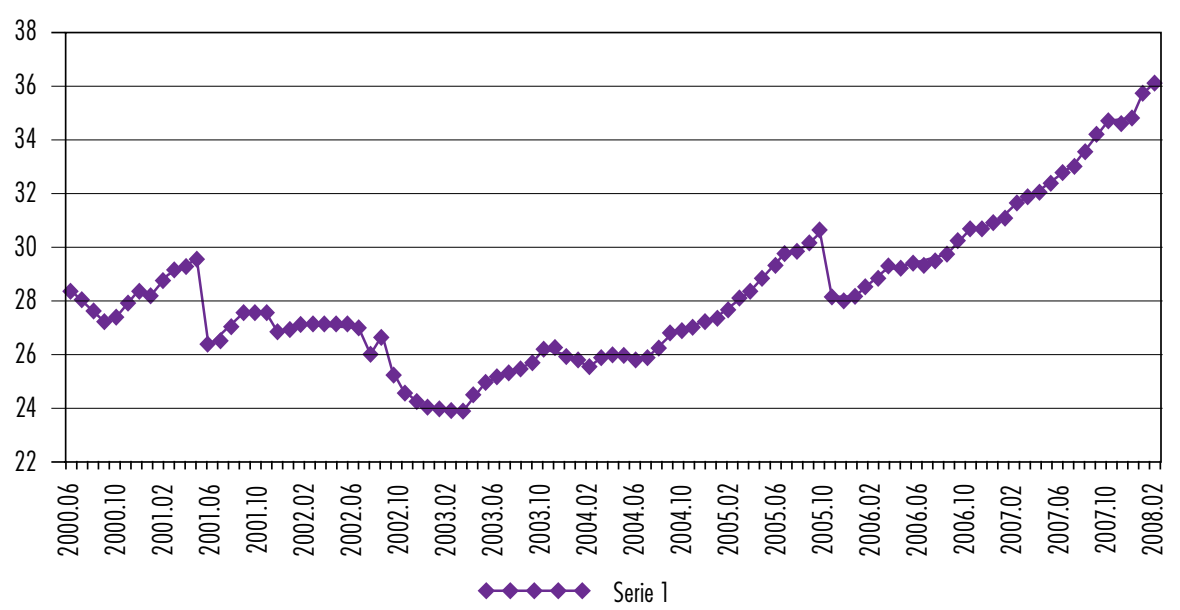

Source: IPEADATA, graphique réalisé par l'auteur.

L'investissement ne reprend donc pas, malgré la diminution des taux d'intérêt et l'accroissement des opérations de crédit dans l'économie brésilienne. La thèse de la financiarisation comme unique responsable ne semble de ce fait plus fournir une explication si pertinente au ralentissement du processus d'accumulation et au faible taux d'investissement observés dans le pays. Ces phénomènes ne peuvent effectivement pas s'appréhender comme des conséquences du poids croissant de la finance et du niveau élevé des taux d'intérêt. S'il est vrai qu'une corrélation entre la baisse du taux d'investissement et l'augmentation des revenus en provenance de la finance est souvent observable, on doit admettre que le lien qui existe entre les deux phénomènes n'est pas la relation de cause à effet établie dans des études précédentes. En effet, depuis le milieu des années 90, les taux d'intérêt ont diminué dans ce pays, ce qui, d'après la thèse énoncée ci-dessus, aurait dû relancer l'investissement. Or, le taux de formation brute de capital fixe continue de stagner (et même de décliner, jusqu'en 2004), alors que c'est justement en ce moment que la finance connait un réel essor.

S'il existe bien un processus de financiarisation de l'économie au Brésil, celui-ci n'est peut-être pas l'unique cause du ralentissement de l'accumulation et des difficultés rencontrées par l'appareil productif. Par ailleurs, ce phénomène de financiarisation s'observe également dans les pays du Centre et ne découle donc pas seulement des spécificités de l'accumulation brésilienne. Il est aussi dû aux difficultés rencontrées dans la sphère productive, notamment à la réduction du taux de profit. 
Jusqu'aux années 1980, la théorie consistant à montrer le lien entre finance et baisse du taux d'investissement visait surtout à démontrer la relation inverse à ce que nous avons étudié jusque là. Pour les auteurs de l'époque, ce n'est pas le poids croissant de la finance qui engendre une diminution ou une stagnation du taux d'investissement, mais plutôt l'amoindrissement des incitations à investir qui stimule la croissance du secteur financier. Ainsi, selon Luiz de Mello Belluzzo (1982: 141), «devant une baisse du taux de profit attendu, pour de nouveaux investissements productifs dans les mêmes secteurs, la masse de profit est orientée vers des investissements financiers et des immobilisations de caractère spéculatif qui, à leur tour, provoquent une réalimentation de l'inflation» ${ }^{4}$.

Pour comprendre la situation actuelle, il semble donc important d'étudier l'évolution du taux de profit. Celle-ci serait-elle responsable d'une stagnation de la formation brute de capital fixe? Les capitalistes préfèreraient ainsi orienter leurs revenus vers des secteurs plus rentables que le secteur productif, ce qui expliquerait l'essor actuel de la finance. En effet, comme nous le révèle la figure 8, le taux de profit de l'appareil productif brésilien ne cesse de décliner depuis la deuxième moitié de la décennie 1970 jusqu'à 1994. Reprenant l'analyse marxiste, nous expliquons cette réduction du taux de profit par celle qu'a subie la productivité du capital depuis la fin du «Miracle économique». Cette perte de productivité du capital, lorsqu'elle s'accompagne d'une moindre hausse de celle du travail, engendre effectivement une baisse du taux de profit (celui-ci correspondant au rapport entre la plus-value réalisée et la somme du travail et du capital utilisé), phénomène évoqué également par l'économiste brésilien Adalmir Marquetti (2004).

\section{Figure 8}

Taux de profit au Brésil (1952-2007)

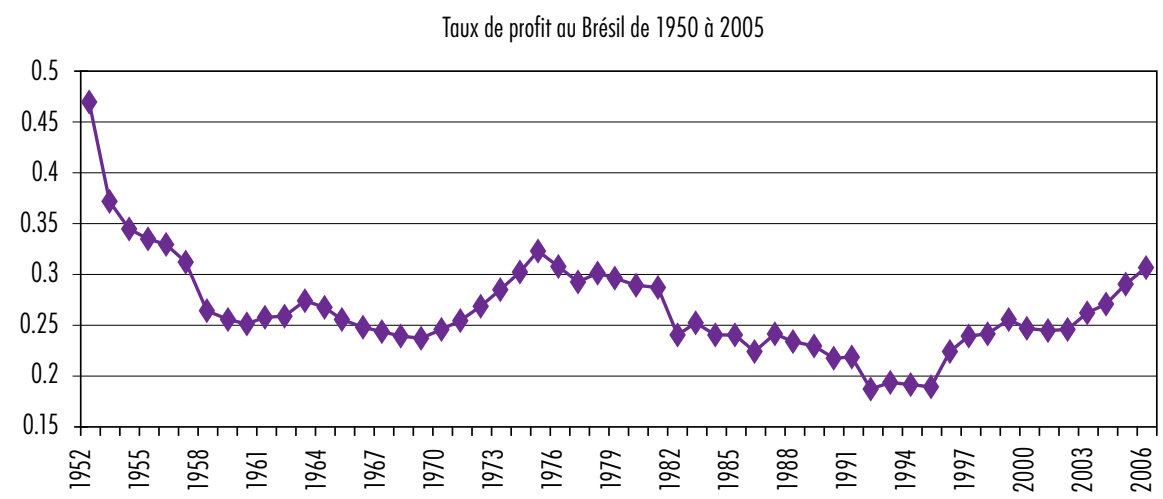

Source: IPEADATA, graphique réalisé par l'auteur. Taux de profit : (PIB-masse salariale) / (Stock de capital fixe + masse salariale).

4 Traduction de l'auteur. 
Pour mieux comprendre le ralentissement du processus d'accumulation, nous reprenons ici la thèse de Grossman (1929) pour qui l'importance de la finance s'explique par la rentabilité extrêmement faible des investissements industriels, le secteur financier fournissant une alternative pour le placement des capitaux excédentaires. De nombreux économistes, en particulier les keynésiens, estiment qu'un abaissement des taux d'intérêt relancerait la croissance: comme le précise Husson (2001: 47), ces auteurs confondent la cause et la conséquence de la crise, l'élévation des taux d'intérêt ayant eu pour fonction de remédier à la faiblesse du taux de profit dans le secteur productif. La baisse du taux de profit dans la sphère productive pousse effectivement les agents économiques à investir chaque jour davantage dans la sphère financière et à devenir plus exigeants quant à la rente recueillie.

\section{L'évolution du taux de profit au Brésil de 1952 à 2007}

Dans cette partie, nous chercherons à montrer le lien qui peut exister entre la théorie sur la baisse tendancielle du taux de profit, et les problèmes que rencontre l'investissement au Brésil. Les données offertes par les offices de statistiques officiels concernent des grandeurs si difficiles à calculer, que nous insistons bien ici sur le fait que les résultats obtenus ne sont que des estimations, et que seule nous importe l'évolution de ces grandeurs. Au Brésil, la composition organique du capital (rapport entre le capital et le travail) n'a pas cessé de s'élever depuis plus de deux décennies. Or, un tel accroissement risque de peser, dans certaines circonstances (notamment s'il est supérieur à l'augmentation du taux d'exploitation $\mathrm{pl} / \mathrm{v})$, sur le niveau du taux de profit correspondant à la formule suivante : $(\mathrm{pl} / \mathrm{v}) /[(\mathrm{c} / \mathrm{v})+1]$, avec $\mathrm{pl} / \mathrm{v}$ comme taux d'exploitation (que nous assimilerons par la suite à la productivité du travail) et $\mathrm{c} / \mathrm{v}$ composition organique du capital.

\section{Calcul du taux de profit}

Pour calculer ce taux de profit qui, selon Karl Marx, correspond à $\mathrm{Pl} /(\mathrm{C}+\mathrm{V})$, nous considérons qu'il est possible de reprendre les données économiques fournies par les offices de statistiques officiels du pays. Pour Marx, le taux de profit se calcule pourtant en valeur, et non en prix. Bien qu'il ne soit pas pertinent de revenir ici sur le débat prix/ valeur lancé depuis le début du vingtième siècle ( $c f$. Duménil, 1980), nous ferons l'hypothèse qu' au niveau national, la somme des prix correspond à la somme des valeurs. Pour notre calcul, le débat sur la transformation de la valeur en prix sera donc mis de côté. Quant aux données utilisées, nous considérons que ce qui se rapproche le mieux de la plus-value chez Marx correspond au PIB auquel on soustrait la masse salariale (chez Marx, la valeur de la production correspond à $\mathrm{Pl}+\mathrm{V}+\mathrm{C}$, avec $\mathrm{Pl}$ comme plus- 
value, $\mathrm{V}$ comme capital variable et $\mathrm{C}$ comme capital constant. $\mathrm{Or}$, le PIB, somme des valeurs ajoutées, ne prend pas en compte les «consommations intermédiaires», que nous pouvons assimiler à $\mathrm{C}$, mais comprend la masse salariale qu'il est donc nécessaire de soustraire pour obtenir la plus-value). En ce qui concerne le capital constant, nous prenons le stock de capital fixe utilisé auquel nous soustrayons l'investissement résidentiel qui, bien que faisant partie de la FBCF, ne participe pas au processus d'accumulation au sein de l'appareil productif. Enfin, pour le capital variable, nous prenons simplement la masse salariale du pays; selon Marx, le capital variable ne correspond pourtant qu'aux seuls travailleurs productifs. Néanmoins, nous ne ferons pas cette distinction, car aussi bien au niveau statistique que théorique, il est extrêmement difficile de distinguer ces travailleurs des improductifs; d'autre part, la masse salariale, relativement au stock de capital fixe, est extrêmement faible, et soustraire les salaires des improductifs ne changerait presque rien.

\section{Evolution de la productivité du capital}

On ne peut analyser la diminution du taux de profit dans un pays, sans y étudier l'évolution de la productivité du travail et du capital. En effet, ces deux variables influent sur la composition organique du capital. Pour les besoins de notre analyse, nous considèrerons que la composition organique correspond à ce que nous appelons généralement intensité capitalistique. Si l'élévation de la productivité du travail dépasse celle du capital, alors la composition organique du capital (ou l'intensité capitalistique) va croître, et réciproquement. Pour faire simple, si l'augmentation de la productivité du travail est supérieure à celle de la productivité du capital, alors le rapport capital sur travail évolue positivement.

D'après les figures $\mathrm{n}^{\circ} 9$ et 10 ci-dessous, la productivité du travail brésilienne connaît essentiellement des périodes d'ascension (hormis de 1952 à 1959, et de 1982 à 1992), alors que la productivité du capital ne cesse de chuter, si ce n'est pour entrer dans une phase de stagnation durant la période du «Miracle économique» d'abord

5 Il pourrait nous être fait le reproche d'utiliser le terme de «productivité du capital », car, selon une approche marxiste, seul le travail est en mesure de créer de la plus-value, et de là, seul le travail est productif. Cependant, pour les besoins de notre analyse, et au risque d'engendrer des querelles sur les termes utilisés, nous passerons outre cette critique. De même, alors que chez Marx, le capital constant correspond aux biens d'équipement et aux matières premières (c'està-dire, à tous les éléments qui sont en mesure de transmettre leur propre valeur à la marchandise sans en produire eux-mêmes), nous ne prendrons ici que le capital fixe, c'est-à-dire les moyens de production matériels qui ne sont pas détruits au cours du processus de production et dont la durée de vie est supérieure à un an. Par cette simplification, nous rejoignons la mesure du taux de profit effectuée par Duménil et Lévy (2002) ainsi que par Moseley (1991) dans le cas des Etats-Unis. 
(de 1966 à 1974), puis de 1992 à nos jours (malgré une légère hausse). Dans ces conditions, on ne peut que comprendre l'accroissement constant de la composition organique observé depuis 1952, le rapport entre le stock de capital fixe et la masse salariale passant de 2 à 5 entre 1952 et 2007 (figure $n^{\circ} 11$ ). Comment expliquer la chute de la productivité du capital au Brésil sur une aussi longue période, de 1950 jusqu'au début des années 1990? Nous présenterons ici les interprétations exposées traditionnellement, puis nous reviendrons par la suite à la théorie de Marx.

Pour Bonelli et Lisboa (2005), la hausse du prix relatif de l'investissement, dont découle la baisse de la productivité du capital, est causée par l'inefficacité de la production nationale de biens d'équipement, mais aussi par le comportement des oligopoles et par l'accroissement des capacités de production oisives. L'inefficacité de l'appareil productif serait donc responsable d'une très forte montée du prix des biens d'équipement comparativement aux prix des biens de consommation. Effectivement, le prix de l'investissement ${ }^{6}$ ne cesse d'augmenter, ce qui fausse d'ailleurs l'étude de l'évolution de la formation brute de capital fixe, comme nous l'avons vu plus haut (figure 1; Feu, 2004b). Le IEDI (2007) révèle ainsi que le prix des biens d'équipement n'a jamais été aussi élevé, ce qui, d'après la thèse de Bonelli, devrait théoriquement engendrer une diminution de la productivité du capital.

Cependant, le lien existant entre la baisse de la productivité du capital et le prix croissant des biens d'équipement est remis en cause par l'évolution récente. Lucilene Morandi (2005) observe comme nous que la productivité du capital connaît un léger redressement depuis le début des années 1990 (figure n¹0). L'explication donnée par Morandi est que l'appréciation de la monnaie brésilienne, qui tend à peser sur le prix des biens d'équipement importés, contribue à élever la productivité du capital, ce que favorise conjointement l'ouverture commerciale grandissante. Cette ouverture, et le surcroît corrélatif d'importation de biens d'équipement, aideraient à remédier au problème d'inefficacité de l'appareil productif national mis en avant dans la théorie de Bonelli. Ces importations permettraient de baisser le prix des biens d'équipement, et empêcheraient donc la perte de productivité du capital. Cependant, comme l'ont montré les travaux du IEDI (2007), les prix des biens d'équipement n'ont jamais été aussi hauts. Le relèvement de la productivité du capital ne peut donc pas s'expliquer par la réduction du prix de l'investissement. Bien que celui-ci soit plus élevé que jamais, le pays connait depuis presque deux décennies la période la plus longue qu'il ait traversée sans que son appareil productif ne soit confronté à une diminution de la productivité du capital.

6 Le prix de l'investissement correspond au rapport entre le prix des biens d'équipement et de l'infrastructure utilisés par l'appareil productif, et le prix des biens de consommation. 
Figure 9

Productivité du travail au Brésil (1952-2007)

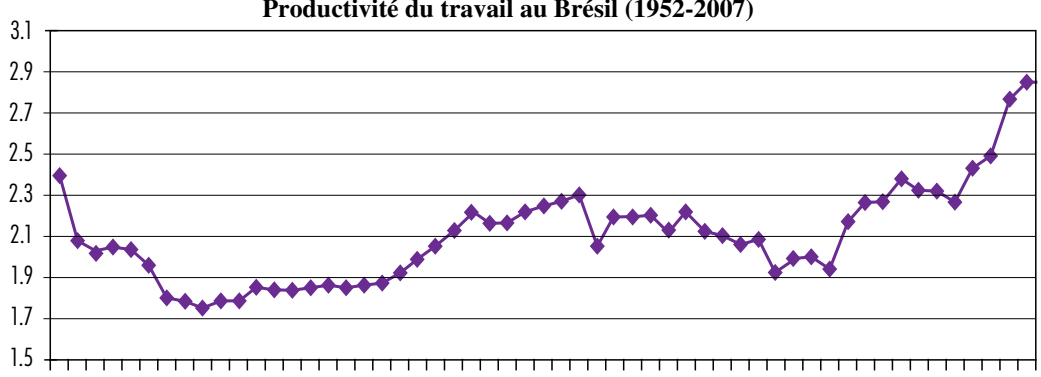

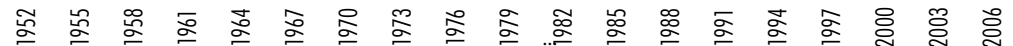

Source: IPEADATA. (Productivité du travail en valeur : PIB/masse salariale.) Graphique réalisé par l'auteur.

Figure 10

Productivité du capital au Brésil (1952-2007)

Productivité du capital au Brèsil de 1950 à 2005

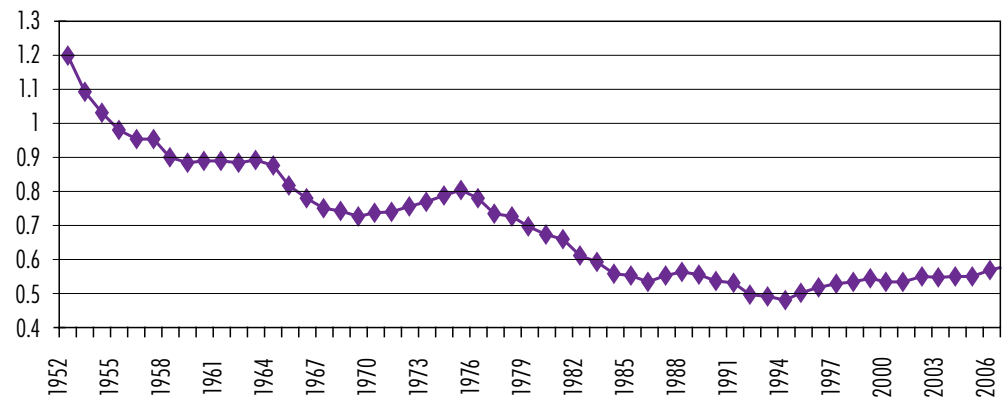

Source: IPEADATA. (Productivité du capital en valeur : PIB/stock de capital fixe.) Graphique réalisé par l'auteur.

Figure 11

Composition organique du capital au Brésil (1952-2007)

Composition organique du capital au Brèsil de 1950 à 2005

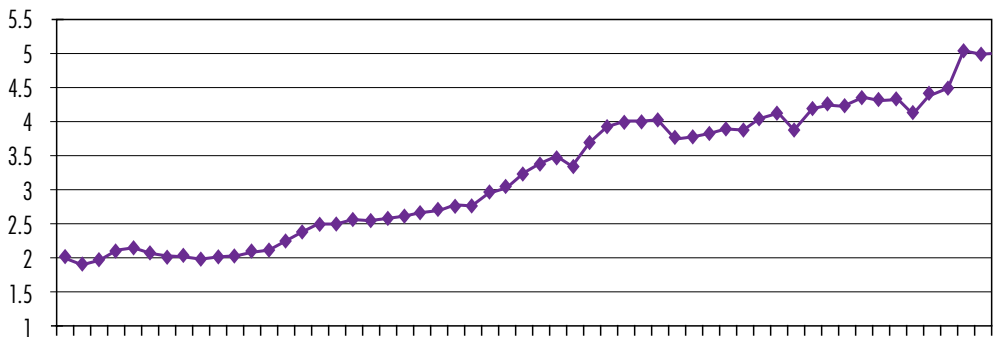

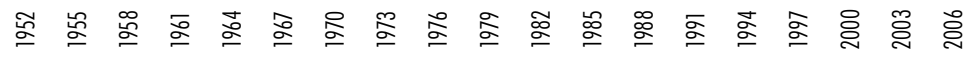

Source: IPEADATA. (Composition organique du capital : Stock de capital fixe/Masse salariale.) Graphique réalisé par l'auteur. 
Figure 12

Prix de l'investissement fixe en biens d'équipement au Brésil, relativement au prix des biens de consommation finale, $1970=1(1970-2005)$

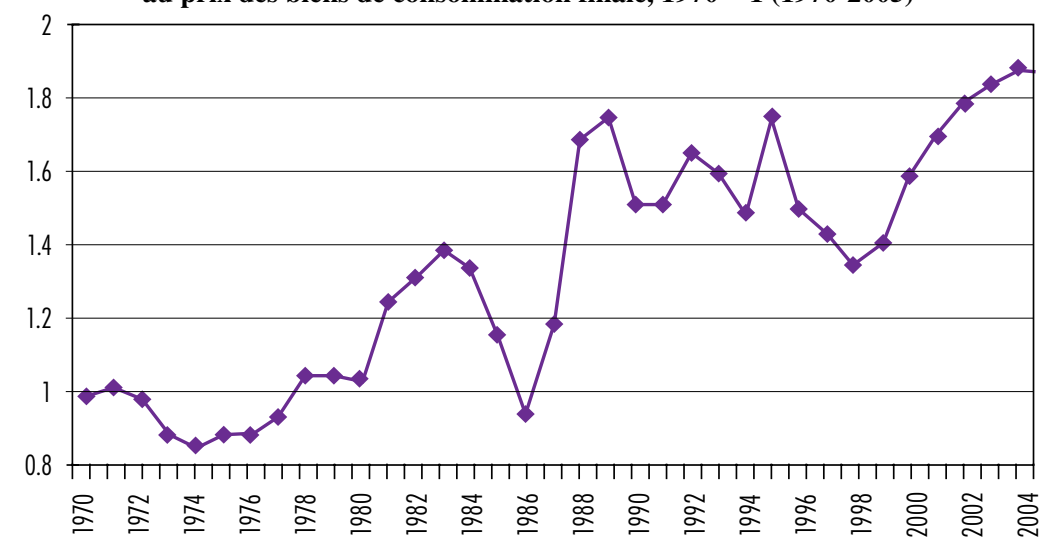

Source: IEDI (2007), graphique réalisé par l'auteur.

Il est donc nécessaire de trouver une nouvelle explication à l'augmentation récente de la productivité du capital. Selon Feu (2004a), cette dernière réside principalement dans le ralentissement du processus d'accumulation. En effet, ce ralentissement implique que les entrepreneurs ne renouvellent pas leurs biens d'équipement, et limitent ainsi l'accroissement du stock de capital fixe (figure 13). Il s'ensuit, certes, une moindre hausse de la valeur ajoutée, mais surtout, le non-renouvellement des biens d'équipement permet d'augmenter fortement la productivité du capital: dans la formule de la productivité du capital, le dénominateur (le stock de capital fixe) s'élève alors moins rapidement que le numérateur (valeur ajoutée).

\section{Figure 13}

Formation brute de capital fixe (FBCF), et FBCF orientée vers l'achat de biens d'équipement, base 10 en 1947 (1947-2007)

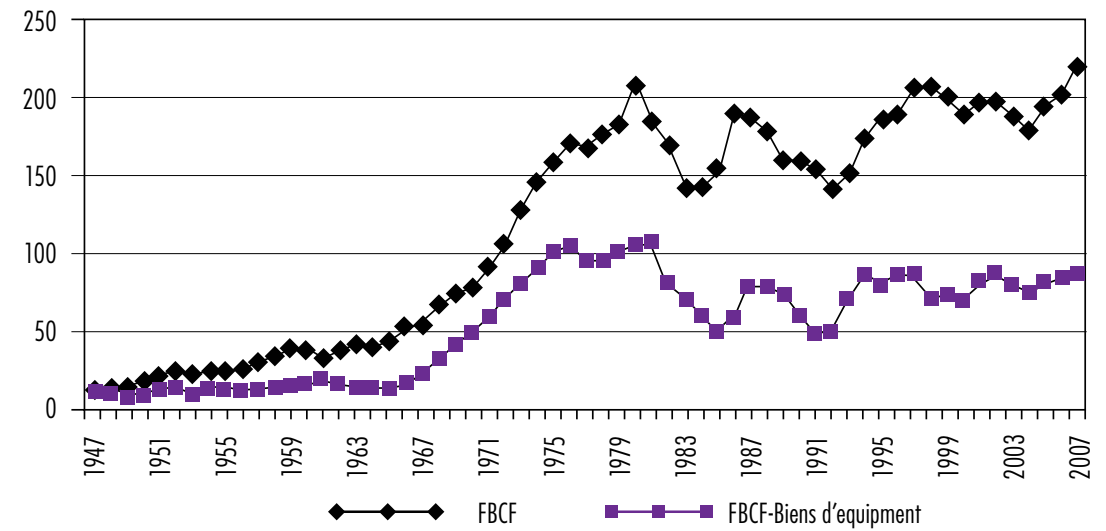

Source: IEDI (2007), graphique réalisé par l'auteur. 
De la même manière, l'une des explications à la réduction récente des inégalités de revenus au Brésil repose sur l'observation d'une plus forte utilisation de main d'œuvre (relativement au capital utilisé) qu'auparavant, notamment dans le secteur formel. D'après l'OCDE ${ }^{7}$, l'élasticité de la demande de main d'œuvre à la croissance est ainsi passée de 0,4 dans la période 1992-1996, à une moyenne de 0,9 entre 1996 et 2004. La productivité du capital peut donc croître, car on observe une substitution du travail au capital qui explique que la valeur ajoutée s'élève plus rapidement que le stock de capital fixe.

La légère élévation de la productivité du capital actuelle s'explique donc par le ralentissement du processus d'accumulation. Inversement, la baisse de cette productivité, de 1950 jusqu'à la décennie 1980, peut se comprendre comme la conséquence d'un taux d'investissement extrêmement important. Du fait de ce fort taux d'investissement, le stock de capital fixe ne cessait d'augmenter et les biens d'équipement étaient sans cesse renouvelés (souvent pour faire face à la concurrence), ce qui pesait fortement sur la productivité du capital, alors qu'au contraire, la productivité du travail était plutôt en hausse.

Nous rejoignons ici la thèse de Marx: lors du processus d'accumulation, de plus en plus de capital constant est utilisé, et ce aux dépens du capital variable, ce qui tend à accroître la composition organique du capital. Effectivement, dans une telle situation, la productivité du capital ne peut que baisser (étant donné qu'on utilise de plus en plus de capital fixe), et la productivité du travail s'élever (on utilise de moins en moins de main d'œuvre).

\section{Une «trajectoire à la Marx» jusqu'en 1994}

Il est reconnu aujourd'hui que depuis le milieu des années 1990 le taux de profit présente une légère ascension au Brésil, alors qu'il n'avait pas cessé de diminuer depuis deux décennies. De nombreuses études, entre autres la thèse de Bruno (2005), cherchent à expliquer ce processus, et insistent en particulier sur le poids croissant de la finance et sur l'augmentation de la productivité du travail. L'accent sera mis essentiellement ici sur la thèse de Miguel Bruno, car il est aujourd'hui l'un des principaux auteurs qui analysent le ralentissement du processus d'accumulation au Brésil sous l'angle du processus de financiarisation et, de plus, en ayant recours à la loi de baisse tendancielle du taux de profit, qu'il met en question pour la période récente.

\footnotetext{
7 OCDE, Employment Outlook (2007: 31).
} 
Selon Miguel Bruno (2005), de 1966 à 1980 l'accumulation brésilienne fut de type profit-led, la hausse du taux de profit entraînant celle du taux d'investissement, contrairement à ce qui se passe depuis la décennie 1990. Sur quoi repose ce taux de profit accru selon Bruno? En réalité, tous les pays situés dans une phase de rattrapage technologique connaissent une telle évolution grâce à la possibilité d'obtenir une élévation temporaire de la productivité du capital, comme l'a montré Johsua (2006) pour l'Europe de l'après-guerre, par exemple.

La hausse du taux de profit durant la période 1966-1974 est engendrée par le progrès technique de l'époque. Ce dernier tend non seulement à élever la productivité du travail, mais il provoque aussi une stagnation de la composition organique du capital. Certes, selon la théorie de Marx sur la baisse tendancielle du taux de profit, l'augmentation de ce taux repose en partie sur celle du taux d'exploitation (productivité du travail), mais il est aussi important d'étudier comment évolue la productivité du capital. Durant la période 1966-1974, au Brésil, l'élévation du taux de profit repose ainsi essentiellement sur une accumulation intensive du capital, la productivité du travail et celle du capital étant toutes deux en ascension.

Dès la fin des années 1970, le taux de profit du Brésil reprend une pente descendante (figure 8). A partir de là, Miguel Bruno ignore une partie de la thèse de Marx, car il ne met plus en avant l'évolution de la productivité du capital. La diminution du taux de profit, expérimentée de 1974 à 1994, serait le résultat direct de l'accumulation intensive de la période antérieure, et des capacités de production oisives qu'elle aurait générées. Effectivement, tandis que les productivités du travail et du capital augmentaient durant cette période de rattrapage technologique, les salaires réels connaissaient un sort différent. La masse salariale, en pourcentage du PIB, n'avait en effet pas cessé de s'amoindrir du milieu des années 1960 jusqu'à la fin des années 1970, passant de 57\% du PIB en 1958 à 44\% en 1980.

Un accroissement des capacités de production oisives est observable dès 1974, phénomène que Bruno considère l'unique raison de l'abaissement du taux de profit observé à la même époque. Ces capacités sont ainsi passées de 10 à 27\% de 1974 à 1983. L'époque du «Miracle économique» (de 1967 à 1974) voit s'amorcer un décalage important entre la production croissante et la demande nationale, les gains de productivité n'étant pas répercutés sur les salaires. Ils permettent au contraire d'élever la plus-value relative, et de profiter de la baisse du prix des biens de consommation (et donc, du moindre coût de reproduction de la force de travail) pour réduire la valeur du capital variable utilisé. Cependant, le volume de la production se trouve dans une période de forte expansion, ce qui, joint à une faible hausse des salaires, est 
à l'origine des phénomènes de surproduction observés dès cette époque. Ces capacités de production oisives tendent à diminuer la plus-value réalisée, et de là, à peser sur le taux de profit. Cependant, ces phénomènes n'expliquent pas à eux seuls la pression exercée sur ce taux.

Dès 1974, la productivité du capital connaît une forte chute que Miguel Bruno peut difficilement expliquer. En réalité, cette chute, qui marque la fin de la période de rattrapage technologique, contribue à élever la composition organique du capital, et donc, à inhiber le taux de profit. Cet accroissement de la composition organique du capital s'explique, selon la thèse de Marx, par la force du processus d'accumulation.

Figure 14

Capacités de production oisives au Brésil, en pourcentage des capacités de production (1970-2007)

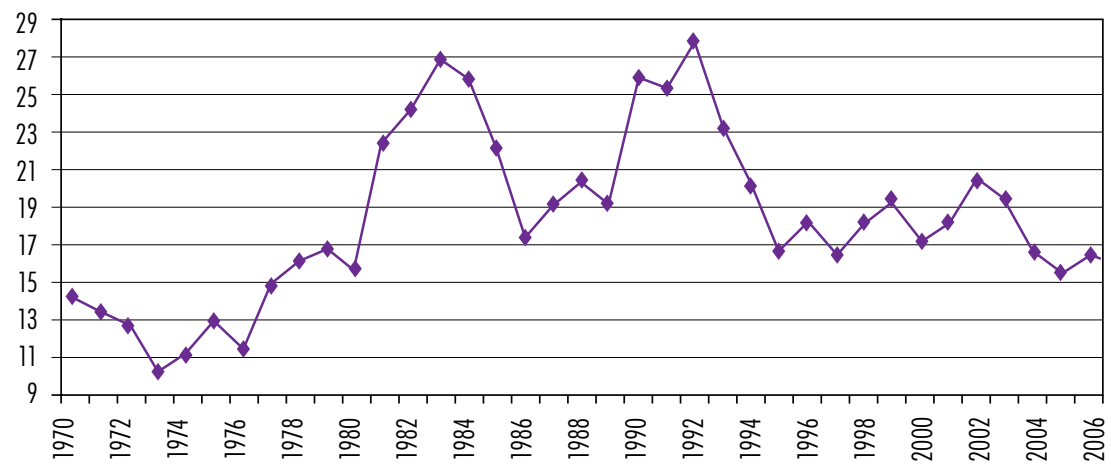

Source: IPEADATA, graphique réalisé par l'auteur.

L'accumulation du capital engendre, par le jeu de la concurrence, une substitution du capital au travail, et par suite, une majeure utilisation du capital constant au détriment des dépenses de capital variable. Or, le capitalisme conduit à la formation d'un appareil productif de plus en plus monopolistique (notamment dans le secteur des biens de production), ce qui explique que même en cas de progrès technique, il est extrêmement difficile d'observer une augmentation de la productivité du capital en dehors d'une période de rattrapage technologique. Ainsi, le prix des biens d'équipement n'a pas cessé de monter depuis la fin des années 1970. Il n'en reste pas moins que Miguel Bruno observe une hausse du taux de profit depuis 1994 au Brésil. Comment expliquer une telle évolution?

Bruno rejoint surtout la thèse régulationniste, développée par des économistes comme Robert Boyer (2000; 2009) ou Michel Aglietta (2007), lorsqu'il analyse 
la situation brésilienne actuelle. Pour lui, comme pour les régulationnistes et certains marxistes ( $c f$. Duménil et Lévy, 2000 ou Chesnais, et al., 2006) qui remettent aujourd'hui partiellement en cause, dans le cas des pays développés, la thèse de Marx sur la baisse du taux de profit, la croissance très instable que connait le Brésil est le résultat d'une «financiarisation de l'économie». L'investissement stagne en raison du surcroît de prélèvements financiers effectués sur le profit brut. Comme dans les pays du Centre, le taux de profit suit l'élévation de la productivité du travail et de l'exploitation engendrée par les revendications croissantes des actionnaires, exploitation favorisée par un marché du travail précaire qui, lui, est le résultat d'un ralentissement de l'investissement et de l'accroissement corrélatif du chômage.

\section{La désindustrialisation comme cause de la hausse du taux de profit depuis 1994}

Selon Miguel Bruno et les partisans de la thèse évoquée ci-dessus, le Brésil ainsi que l'ensemble des pays du Centre, ne suivraient plus une «trajectoire à la Marx». Effectivement, la hausse du taux de profit actuelle remettrait en cause la thèse de Marx sur la baisse tendancielle du taux de profit. Les spécialistes de la question insistent souvent sur le terme tendancielle pour expliquer que Marx ne voyait pas dans la diminution du taux de profit une constante du mode de production capitaliste. Il est pourtant nécessaire de s'interroger sur les éléments qui expliquent les renversements de tendance. Son augmentation actuelle peutelle être expliquée par la lecture du Capital?

Dans l'analyse régulationniste, la variation de la productivité du capital n'est évoquée que dans les périodes où le taux de profit s'amoindrit. Dès la fin des années 1960, la chute de la productivité du capital aurait provoqué une élévation de la composition organique dans les pays du Centre, ce qui, avec le ralentissement de la croissance de la productivité du travail, serait à l'origine de la réduction du taux de profit observée jusqu'au début des années 1980. Rapidement, les pays du Centre, ainsi que le Brésil à partir des années 1990, seraient entrés dans une phase de financiarisation de leur économie qui devait permettre d'élever la productivité du travail, et de là, le taux de profit. Les pays du Centre ainsi que le Brésil se situeraient aujourd'hui dans cette phase caractérisée par un redoublement de productivité du travail. Cependant, aussi bien dans l'analyse de Bruno que dans celle des régulationnistes, l'évolution actuelle de la composition organique du capital n'est plus présentée comme l'une des composantes du taux de profit. Or, il est nécessaire d'analyser la croissance de la productivité du capital au regard de celle du travail pour évaluer la composition 
organique du capital, qui est aussi importante que la productivité du travail dans la mesure du taux de profit.

La figure $n^{\circ} 10$ montre que la productivité du capital ne diminue plus et serait même légèrement en hausse depuis le début de la décennie 1990. De même, la productivité du travail, après avoir stagné durant toute la décennie 1980, connaitrait aujourd'hui une forte ascension. Malgré celle-ci, en raison de l'accroissement de la productivité du capital, la composition organique du capital augmente beaucoup moins rapidement depuis la décennie 1980 que durant les quelques années suivantes le «Miracle économique». Ainsi, de 1983 à 2005, la composition organique a crû deux fois moins vite que durant la période 1963-1983. Comment expliquer une telle situation?

On peut distinguer deux périodes. De 1983 à 1994, la stagnation de la composition organique du capital s'accompagne d'une diminution de la productivité du travail, et donc, d'une baisse du taux de profit. Ces deux phénomènes s'expliquent par la situation de crise que traverse le Brésil des années 1980, notamment par les difficultés rencontrées pour rembourser sa dette externe. Face à ces difficultés accrues de financement, le taux d'investissement chute brusquement durant cette période, ce qui implique que les biens d'équipement utilisés par l'appareil productif sont peu renouvelés. Le volume de la production stagne également, mais dans une moindre proportion. En conséquence, la productivité du capital cesse de baisser, et commence même à remonter dès 1991. Par contre, dès 1985, et ce jusqu'en 1994, la productivité du travail diminue, et avec elle le taux de profit, par suite de ces limitations rencontrées par l'appareil productif, d'autant plus que les luttes sociales de l'époque font y maintenir un volume de main d'œuvre relativement élevé (ce qui explique que la part de la masse salariale dans le PIB ne cesse de croître durant toute la décennie 1980).

Durant la deuxième période étudiée, de 1994 à aujourd'hui, la composition organique du capital continue de stagner (à l'exception d'un redressement notable, entre 2002 et 2005), alors que la productivité du travail s'élève fortement : l'écart croissant entre les deux grandeurs engendre alors une ascension du taux de profit. Comment expliquer ces phénomènes? En ce qui concerne le faible accroissement de la composition organique, on peut y voir la conséquence de l'augmentation de la productivité du capital, qui peut être expliquée de la même manière que pour la période précédente: selon le IEDI, depuis la décennie 1990 la productivité du capital est sur une pente ascendante car les machines et biens d'équipement ne sont pas renouvelés du fait de la «désindustrialisation relative» (IEDI, 2005). Quant à l'élévation de la productivité du travail, nous allons voir maintenant que ce n'est pas tant une conséquence des exigences de la finance, que le résultat de cette désindustrialisation. 
D'après l'analyse régulationniste, les exigences des financiers (qui se manifestent au Brésil, selon Bruno, par le maintien de taux d'intérêt très élevés) sont néfastes pour l'investissement, et les entreprises se voient alors contraintes, sous cette pression, de peser fortement sur les salaires. La financiarisation de l'économie et les exigences accrues des financiers peuvent-elles être à l'origine de l'augmentation de la productivité du travail, comme l'affirment aussi bien les régulationnistes que Miguel Bruno? Les taux d'intérêt réels sont moins hauts aujourd'hui qu'à la fin de la décennie $1980^{8}$, et pourtant la productivité du travail ne s'est redressée qu'à partir de 1994 alors qu'elle n'avait pas cessé de chuter depuis 1985. Il faut donc trouver une autre explication à cette évolution.

Il semble également que la thèse régulationniste devrait pouvoir être confirmée en partie par l'observation d'une accentuation importante des inégalités, si l'élévation de la productivité du travail était la conséquence immédiate de la financiarisation de l'économie. Or, depuis le milieu de la décennie 1990 les inégalités diminuent dans ce pays, notamment du fait d'une réduction de l'écart existant entre les salaires des plus qualifiés et ceux des moins qualifiés9. L'indice Gini est ainsi passé, entre 1994 et 2008, de 0,60 à 0,56 - alors qu'il n'avait pas cessé d'augmenter durant les décennies précédentes. Cette baisse découle du processus de désindustrialisation entamé par le Brésil depuis le milieu des années 90 (Gaulard, 2008). C'est en fait cette particularité du Brésil actuel, et non les pressions de la finance, qui entraîne par ailleurs un accroissement de la productivité du travail. En effet, c'est l'utilisation plus importante de travail non qualifié -dont la rémunération est évidemment moins élevée que celle du travail qualifié-, qui explique qu'alors que les inégalités se réduisaient au Brésil, la part des salaires dans le revenu national a fortement chuté depuis la décennie 1990 (figure 15).

L'explication n'est donc pas aussi simple que celle proposée par les régulationnistes ou les marxistes qui minimisent la portée de la thèse de Marx sur la baisse tendancielle du taux de profit. Ce n'est pas le secteur financier qui bloque la reprise

8 En 1995, le taux d'intérêt réel de court terme atteint $21 \%$ en 1990 , contre $4,60 \%$ en janvier 2010.

9 Selon le IEDI, le pays est entré dans une phase de «désindustrialisation relative» qui se caractérise par une diminution de la part des biens à fort contenu technologique, nécessitant l'utilisation de main d'œuvre qualifiée, dans la production industrielle nationale. Le Brésil se spécialise en effet dans des secteurs intensifs en ressources naturelles (chimie, industrie métallurgique et sidérurgie...) et en main d'œuvre non qualifiée, et abandonne progressivement les secteurs traditionnels (textile, électronique...) (IEDI, 2005). Alors que les secteurs intensifs en ressources naturelles constituaient 35,9\% de la production industrielle en 1991, cette part s'élève à 45,7\% en 2009. 
Figure 15

Masse salariale brésilienne en pourcentage du PIB (1952-2008)

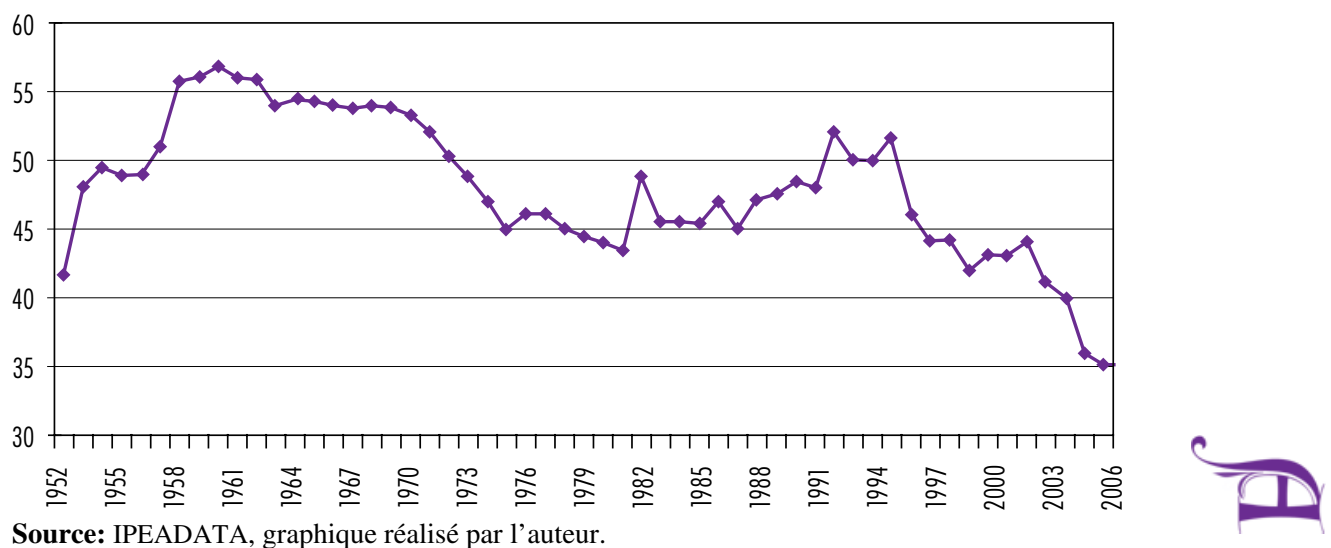

du processus d'accumulation. Cette accumulation fut freinée dans les années 1980, aussi bien au Brésil que dans les pays du Centre, par les difficultés rencontrées dans l'appareil productif. l'affaiblissement du taux de profit ne permettait plus aux Etats et aux entreprises de faire face à leurs engagements financiers qui avaient été pourtant jusque là indispensables à l'investissement. La situation actuelle de ralentissement du processus d'accumulation semble avoir résolu le problème en stimulant une nouvelle hausse du taux de profit: cette dernière n'est donc pas le reflet d'une stimulation du capital pourtant indispensable au développement du mode de production capitaliste.

\section{Conclusion}

Pour conclure, il est donc nécessaire d'insister sur le fait que les raisons du blocage du processus d'accumulation au Brésil ne se situent pas à l'extérieur de l'appareil productif, dans une quelconque sphère financière, mais elles seraient plutôt ancrées dans ce même processus d'accumulation. Cette thèse s'oppose ainsi aux explications les plus répandues de la désindustrialisation brésilienne, avancées aussi bien par des économistes régulationnistes que marxistes, qui rendent la finance responsable des difficultés rencontrées par les économies capitalistes.

Au contraire, dans le cas du Brésil le développement de la finance permet de remédier en partie à ces blocages, notamment en offrant un nouveau secteur suffisamment rémunérateur aux capitalistes, et pourrait même expliquer l'élévation du taux de profit dont bénéficie le Brésil depuis 1994. En effet, depuis le milieu de la décennie 
1990, la productivité du capital augmente légèrement en raison du processus de «désindustrialisation relative» expérimenté depuis lors par le pays, cette désindustrialisation se caractérisant par une moindre utilisation de capital fixe au profit du travail, ainsi que par un accroissement de la productivité du travail. Cet article rejoint ainsi la thèse de Karl Marx sur la baisse tendancielle du taux de profit et sur les difficultés inhérentes au système capitaliste, les blocages que celui-ci rencontre inéluctablement. Si la diminution du taux de profit est contrecarrée depuis 15 ans au Brésil, le ralentissement du processus d'accumulation qui permet un tel processus pourrait pourtant bientôt remettre en cause la place de ce pays sur la scène internationale.

Ainsi, la désindustrialisation pourrait être dangereuse pour le Brésil qui risque de perdre une place importante dans les relations commerciales internationales. L'instabilité du cours des matières premières, accentuée par la récente crise, ainsi que les difficultés croissantes pour trouver des sources de financement, mettent actuellement de plus en plus en question la pertinence de l'orientation prise par l'appareil productif brésilien. Néanmoins, un profond dilemme demeure, car non seulement une relance du processus d'accumulation risquerait de peser sur le taux de profit, mais surtout elle accentuerait à nouveau les inégalités entre travailleurs qualifiés et non qualifiés. D’où la légitimité de nous interroger sur les capacités du mode de production capitaliste à maintenir une croissance économique durable et acceptée par tous...

\section{Bibliografía}

Aglietta, Michel y Laurent Berrebi, Désordres dans le Capitalisme mondial, Odile Jacob, Paris, 2007.

Bonelli, Regis e Edmar Lisboa Bacha, «Uma interpretação das causas da desaceleração econômica do Brasil», Revista de Economia Política, São Paulo, vol. 25, n³, julhosetembro, 2005, pp. 163-189.

Boito Junior, Armando, «A burguesia no goberno Lula», en Eduardo Basualdo y Enrique Arceo, Neoliberalismo y sectores dominantes, Tendencias globales y experiencias nacionales, Buenos Aires, CLACSO, 2006.

Boyer, Robert, «Feu le Régime d'accumulation tiré par la finance», Revue de la Régulation, $\mathrm{n}^{\circ} 5$, 1er semestre de 2009, http://regulation.revues.org/index 7367.html

, «Is a finance led growth regime a viable alternative to fordism? A preliminary analysis», Economy and Society, vol. 29(1), 2000.
Brender, Anton et Florence Pisani, Les Déséquilibres internationaux, Repères, La Découverte, Paris, 2007.

Bresser Pereira, Luis, Macroeconomia da estagnação: crítica da ortodoxia convencional no Brasil pós-1994, São Paulo, Editora 34, 2007.

Bruno, Miguel, «Financiarisation et Accumulation du capital productif au Brésil», Revue Tiers Monde, Paris, $\mathrm{n}^{\circ} 189$, janvier-février, 2007.

, Les Transformations du régime d'accumulation au Brésil à la lumière de la Théorie de la Régulation, Paris, Thèse de Doctorat, 2005.

Chesnais, François, Suzanne de Brunhoff, Gérard Duménil, Michel Husson, La Finance capitaliste: Séminaire d'études marxistes, PUF, Paris, 2006. 
Duménil, Gérard, De la Valeur aux prix de production, une réinterprétation de la transformation, Economica, Paris, 1980.

Duménil, Gérard, Dominique Lévy, «The profit rate: where and how much did it fall? Did it recover? (USA 1948-2000)», Review of Radical Political Economics, vol. $34, \mathrm{n}^{\circ} 1,2002$, pp. 437-461.

Feu, Aumara, «Avaliação da produtividade de capital no século XX», Economia e Energia, São Paulo, n 43, março-abril, 2004a.

, «Evolução do investimento no Brasil», Economia e Energia, São Paulo, n ${ }^{\circ} 46$, outubro-novembro, 2004b.

Gaulard, Mylène, Accumulation du Capital et inégalités: une approche comparée Chinel Brésil, Paris, PhD Thesis, Panthéon-Sorbonne, 2008.

Gonçalves, Reinaldo, «Desestabilização macroeconômica e dominação do capital financeiro no Brasil», en Basualdo M., Arceo E., Neoliberalismo y sectores dominantes, Tendancias globales y experiencias nacionales, Buenos Aires, CLACSO, 2006.

Grossman, Henryk, La Ley de acumulación y del derrumbe del sistema capitalista, México, Siglo XXI editores, 1979.

Husson, Michel, «Années 70: La crise et ses leçons», en Michel Husson, Isaac Johsua, Eric Toussaint, Michel Zerbato, Crises Structurelles et financières du capitalisme au 20e siècle, Paris, Syllepse, 2001.

IEDI, «O problema no preço relativo do investimento fixo no Brasil», São Paulo, agosto, 2007.
, «Ocorreu uma desindustrializacão no Brasil», São Paulo, novembro, 2005.

Johsua, Isaac, Une Trajectoire du capital: de la crise de 1929 à celle de la nouvelle économie, Paris, Editions Syllepse, 2006.

Marquetti, Adalmir, «A economia brasileira no capitalismo neoliberal: progreso técnico, distribuição, crescimento e mudança institucional», março, 2004, www.sep.org.br

Morandi, Lucilene, «Estoque e produtividade do capital fixo: Brasil 1950-2004», Textos Para Discussão $n^{\circ}$ 174, Rio de Janeiro, Universidade Federal Fluminense, agosto, 2005.

Moseley, Fred, The Falling Rate of Profit in the Postwar United States economy, St Martin's Press, New York, 1991.

Salama, Pierre, «Do produtivo ao financeiro e do financeiro ao produtivo na Ásia e na América Latina», Revista da Sociedade Brasileira de Economia Política, $\mathrm{n}^{\circ}$ 6, Rio de Janeiro, junho, 2000.

Serra, José, Desenvolvimento capitalista no Brasil, São Paulo, Editora Brasiliense, 1982.

Stockhammer, Engelbert, «Financialisation and the slowdown of accumulation», Cambridge Journal of Economics, vol. 28(5), 2004, pp. 719-741.

Teixeira Torres Filho, Ernani y Fernando Puga Pimentel, «Investimento na economia brasileira: o caminho do crescimento sustentado», Perspectiva do investimento 2007/2010, São Paulo, BNDES, junho, 2007. 
El Instituto de Investigaciones Económicas de la Universidad Nacional Autónoma de México

$\mathrm{y}$

La Comisión Económica para América Latina y el Caribe

de la Organización de las Naciones Unidas

CONVOCAN AL

PREMIO INTERNACIONAL DE INVESTIGACIÓN EN DESARROLLO ECONÓMICO

\section{JUAN F. NOYOLA}

\section{CONSIDERANDO}

- que entre las finalidades de la Universidad Nacional Autónoma de México está la de promover el estudio de los problemas económicos que atañen en general al mundo y a la región de América Latina y México en particular;

- que uno de los objetivos del Instituto de Investigaciones Económicas es fomentar el análisis económico y la propuesta de alternativas de solución;

- que el desarrollo económico es un concepto que abarca el crecimiento económico pero también la búsqueda de la igualdad y la justicia social así como la sustentabilidad y la equidad de género entre otros objetivos sociales y económicos para las naciones y los ciudadanos del mundo entero. - que la CEPAL tiene como propósito impulsar y promover el desarrollo económico y social sustentable en América Latina y el Caribe, tanto en lo que se refiere a colaborar hacia un mejor diseño, instrumentación y evaluación de políticas públicas como en impulsar la reflexión e intercambio de ideas en torno al desarrollo económico y social, identificar sus determinantes y las formas de superar sus obstáculos

- que Juan F. Noyola fue un distinguido economista mexicano, egresado de la Universidad Nacional Autónoma de México y funcionario de la CEPAL entre 1950 y 1960 ;

- que se instituyó el Premio Internacional de Investigación en Desarrollo Económico que lleva el nombre de Juan F. Noyola;

- que el Premio consiste en un incentivo económico y un reconocimiento académico a las mejores investigaciones sobre desarrollo económico en América Latina.

El Instituto de Investigaciones Económicas (IIEc) y la Comisión Económica para América Latina y el Caribe (CEPAL) convocan al Premio Internacional de Investigación en Desarrollo Económico Juan F. Noyola, versión 2010.

\section{PREMIOS}

Primer lugar: 100000.00 pesos mexicanos o su equivalente en dólares estadounidenses y diploma.

Segundo lugar:

30000.00 pesos mexicanos o su equivalente en dólares estadounidenses y diploma.

\section{BASES}

1. Podrán participar investigadores y estudiosos que sean ciudadanos o residentes latinoamericanos.

2. No podrán participar investigadores o académicos, ni, personal que labore en el Instituto de Investigaciones Económicas de la Universidad Nacional Autónoma de México o en la Comisión Económica para América Latina y el Caribe.

3. Las investigaciones deberán abordar temas relativos al desarrollo económico, sobre aspectos teóricos o empíricos y contribuir al análisis y formulación de alternativas para su solución, de acuerdo con los considerandos de la presente convocatoria.

4. Se tomará en cuenta el rigor científico y metodológico del análisis y el sustento bibliohemerográfico, estadístico o de investigación de campo.

5. Los trabajos deberán ser originales e inéditos, no estar pendientes de resolución en ningún otro certamen, ni haber sido elaborados por encargo específico remunerado. No podrán participar trabajos que hayan sido premiados en cualquier otro certamen.

6. Las investigaciones podrán ser individuales, colectivas o tesis de posgrado. (No se aceptarán tesis de licenciatura.) Por investigaciones colectivas se entienden aquellas que guardan unidad en su conjunto y entre sus partes y que presentan en colaboración dos o más autores, y no la suma de artículos independientes entre sí de diferentes autores.

7. Cada autor podrá concursar solamente con un trabajo. 
8. Los trabajos podrán presentarse en español o en portugués, tendrán una extensión máxima de 200 cuartillas tamaño carta a doble espacio escritas a 12 puntos por una sola cara (1 700 caracteres por cuartilla aproximadamente). Se presentarán en versión Windows 97 y las tablas, mapas o cualquier tipo de anexo que pueda modificarse, deberá presentarse como imagen o fotografía, para el caso de los trabajos recibidos en disco compacto.

9. Los participantes que residan en la Ciudad de México entregarán siete ejemplares del trabajo, empastados o engargolados; firmados con seudónimo, en la primera hoja del trabajo deberá anotarse el nombre del trabajo, el seudónimo y el nombre del Premio. Además se entregarán, al mismo tiempo y por separado: siete ejemplares del resumen de 10 cuartillas máximo; un sobre cerrado en donde se incluirá, en la parte exterior el título del trabajo, el seudónimo y nombre del Premio. En el interior del sobre deberán venir el seudónimo, nombre, domicilio, teléfono, currículum vitae (resumido) del autor o los autores, título del trabajo y correo electrónico. Por ningún motivo deberá aparecer el nombre del autor o autores en el trabajo, si apareciera(n) el trabajo será descalificado de inmediato. Los sobres de las investigaciones premiadas serán abiertos por el jurado una vez terminada la evaluación correspondiente.

10. Los participantes que radiquen en la República mexicana o en el extranjero deberán enviar por mensajería especializada un sobre que contenga un disco compacto con dos archivos, el primero deberá corresponder al trabajo cubriendo todas las especificaciones antes mencionadas, el segundo archivo será un resumen de 10 cuartillas máximo, además en el mismo paquete se enviará un sobre cerrado en donde se incluirá, en la parte exterior el título del trabajo, el seudónimo y nombre del Premio. En el interior del sobre deberán venir el seudónimo, nombre, domicilio, teléfono, currículum vitae (resumido) del autor o los autores, título del trabajo, correo electrónico y país de residencia, es muy importante anotar y destacar el número telefónico donde se le pueda localizar durante los siguientes ocho meses aproximadamente. Por ningún motivo deberá aparecer el nombre del autor o autores en el trabajo, si apareciera(n) el trabajo será descalificado de inmediato. Los sobres de las investigaciones premiadas serán abiertos por el jurado una vez terminada la evaluación correspondiente.
11. La recepción de los trabajos inicia el día siguiente de la publicación de esta convocatoria, de las 9:00 a las 17:00 horas, de lunes a viernes y vence el martes 5 (cinco) de octubre de 2010 a las 14:00 horas (equivalente a seis meses posteriores al lanzamiento de la convocatoria). Los trabajos se recibirán en la Secretaría Académica del Instituto, Circuito Mario de la Cueva $\mathrm{s} / \mathrm{n}$, edificio A, segundo piso, corredor derecho, Ciudad Universitaria, 04510, México, D. F., tels. 56230141,56230127 y 56230101 . Durante el periodo vacacional de verano (del 5 al 23 de julio de 2010), no se recibirán trabajos. En caso de que la documentación se envíe por correo certificado se tomará en cuenta la fecha del matasellos.

12. Los trabajos que no cumplan con lo dispuesto en estas bases serán excluidos del certamen.

13. El jurado calificador estará integrado por el(a) Director(a) del Instituto de Investigaciones Económicas de la UNAM, quien lo presidirá, por el coordinador del Premio, así como por cinco especialistas de reconocido prestigio, tres serán designados por el Instituto de Investigaciones Económicas y dos designados por la Comisión Económica para América Latina y el Caribe

14. La decisión del jurado será inapelable y podrá declarar desierto el Premio en alguno o en ambos lugares.

15. El Instituto publicará el trabajo merecedor del primer lugar. Los trabajos publicados se sujetarán a las disposiciones de la UNAM en materia de derechos de autor.

16. Los trabajos no ganadores así como los sobres correspondientes cerrados se destruirán una vez que el jurado haya tomado su decisión. En consecuencia, no se devuelven.

17. Los puntos no previstos en esta convocatoria serán resueltos por el jurado.

\section{PREMIACIÓN}

Los premios y diplomas serán entregados en ceremonia especial que se celebrará en el Instituto de Investigaciones Económicas, en 2011.

A te n t a mente, "POR MI RAZA HABLARÁ EL ESPÍRITU" Ciudad Universitaria, D. F., 5 de abril de 2010.

EL DIRECTOR

DR. JORGE BASAVE KUNHARDT
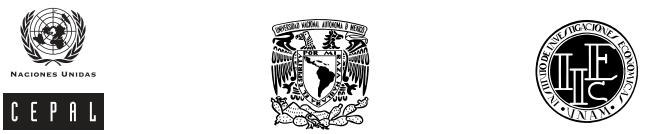

100 vงмm

Consulte la presente convocatoria en:

http://www.iiec.unam.mx http://www.cepal.org.mx http://www.cepal.org 